\title{
用于高精度超远程激光测距的高光束质量大能量 皮秒激光器
}

㚞仲维 $1,2,3^{*}$, 黄玉涛 ${ }^{1,2,3}$, 间晓超 ${ }^{1,2}$, 郭广妍 ${ }^{1,2}$, 白振㤁 ${ }^{1,2}$, 何建国 ${ }^{1,2,3}$, 赵天卓 ${ }^{1,2}$, 张鸿博 $1,2,3$

1. 中国科学院光电研究院, 北京 100094 ;

2. 国家半导体百浦激光工程技术研究中心, 北京 100094 ;

3. 中国科学院大学, 北京 100049

* 联系人, E-mail: fanzhongwei@aoe.ac.cn

\section{A high-beam-quality, high-pulse-energy picosecond laser system for high precision ultra-remote laser ranging}

\author{
Zhongwei Fan $^{1,2,3^{*}}$, Yutao Huang ${ }^{1,2,3}$, Xiaochao Yan ${ }^{1,2}$, Guangyan Guo ${ }^{1,2}$, Zhen'ao Bai ${ }^{1,2}$, \\ Jianguo He ${ }^{1,2,3}$, Tianzhuo Zhao ${ }^{1,2}$ \& Hongbo Zhang ${ }^{1,2,3}$ \\ ${ }^{1}$ Academy of Opto-Electronics, Chinese Academy of Sciences, Beijing 100094, China; \\ ${ }^{2}$ National Engineering Research Center for DPSSL, Beijing 100094, China; \\ ${ }^{3}$ University of Chinese Academy of Sciences, Beijing 100049, China \\ * Corresponding author, E-mail: fanzhongwei@aoe.ac.cn \\ doi: 10.1360/N972019-00041
}

激光超远程测距是从地面台站发射窄脉宽高能量激光 束对 30 万千米以上的探测目标(月球和探月飞行器)进行高 精度测距的一种直接测距方法. 激光超远程测距不仅能为 各类探月飞行器进行精确定轨和变轨提供重要指导数据, 还可以为天文地球动力学、地月科学、月球物理学和引力 理论等研究所需的高精度月球距离提供数据基础. 目前, 国际上已实现厘米量级月球激光测距精度. 2018年1月, 中 国科学院云南天文台使用大能量纳秒激光器在国内首次 实现测距精度优于 $1 \mathrm{~m}$ 的月球激光超远程测距. 表 1 是国际 天文台站用于月球测距所配置的大脉冲能量激光源及获 得的测距精度. 因此, 研制大能量高光束质量皮秒激光器 对全面提升我国高精度超远程激光测距技术水平具有重 要的现实意义.

中国科学院光电研究院基于自行研制的 $15 \mathrm{~mm}$ 大口径 侧蒠模块, 实现了重复频率 $100 \mathrm{~Hz}$ 、脉宽 $70 \mathrm{ps}$ 、单脉冲能 量 $330 \mathrm{~mJ}$ 、光束质量 $M^{2}<2.5$ 的皮秒激光输出. 该皮秒激光 采用“锁模振荡+再生放大+能量放大”的主振荡功率放大 (master oscillator power amplifier, MOPA)结构, 如图1(a)所 示, 同时实现了大能量和高光束质量的激光输出. 锁模振 荡器输出的重复频率 $80 \mathrm{MHz}$ 、脉冲宽度 $70 \mathrm{ps}$ 、单脉冲能量
表 1 各天文台站用于激光测月系统激光光源的主要参数及测 距精度

Table 1 Main parameters of laser sources and accuracy of observation at lunar laser ranging stations

\begin{tabular}{|c|c|c|c|c|}
\hline $\begin{array}{l}\text { 测月 } \\
\text { 台站 }\end{array}$ & $\begin{array}{c}\text { 脉冲 } \\
\text { 宽度(ps) }\end{array}$ & $\begin{array}{c}\text { 脉冲 } \\
\text { 能量 }(\mathrm{mJ})\end{array}$ & $\begin{array}{c}\text { 重复 } \\
\text { 频率 }(\mathrm{Hz})\end{array}$ & $\begin{array}{c}\text { 测距 } \\
\text { 精度 }(\mathrm{cm})\end{array}$ \\
\hline McDonald & 200 & 1500 & 10 & $\sim 3.5$ \\
\hline Grasse & 150 & 200 & 10 & $<3$ \\
\hline APOLLO & 90 & 115 & 20 & $\sim 0.5$ \\
\hline Wettzell & 10 & $\begin{array}{c}130 \\
\text { (双脉冲) }\end{array}$ & 400 & $\sim 0.5$ \\
\hline Matera & 50 & 100 & 10 & $\sim 2$ \\
\hline 云南天文台 & 10000 & 3000 & 10 & $<100$ \\
\hline
\end{tabular}

$2 \mathrm{~nJ}$ 的1064 nm激光经过再生放大器选单和放大后, 获得重 复频率 $100 \mathrm{~Hz}$ 、脉冲能量 $2.3 \mathrm{~mJ}$ 的激光输出, 随后进入能量 放大器进行放大. 为了避免元器件损伤和降低非线性效应, 采用逐渐扩束的方式, 依次进入 $6.35 \mathrm{~mm}$ 口径侧原 $\mathrm{Nd}$ : $\mathrm{YAG}$ 模块和两个 $15 \mathrm{~mm}$ 口径侧百 $\mathrm{Nd}: \mathrm{YAG}$ 模块进行双程放大.

大口径侧原模块是大能量皮秒激光系统的关键部件, 
国外厂商对于口径 $12 \mathrm{~mm}$ 以上的侧泵模块对我国实行长期 禁运. 中国科学院光电研究院经过攻关, 采用全国产化器 件成功研发出具有完全自主知识产权的 $15 \mathrm{~mm}$ 大口径侧洜 Nd:YAG模块, 如图1(b)所示. 该模块采用15维环形泵浦和 放大自发辐射(amplified spontaneous emission, ASE)抑制技 术, 获得了高于 $90 \%$ 均匀性的储能分布, 在 $100 \mathrm{~Hz}$ 重复频 率下获得储能 $2.5 \mathrm{~J}$ 、小信号增益 5 的高能量输出, 如图 $1(\mathrm{c})$ 所示. 模块具有很好的泵浦均匀性, 实现 $90 \%$ 口径内PV值
小于 $10 \%$ 的高均匀葲浦分布，如图1(d)所示. 该大口径侧 泵放大模块用于两级双通能量放大，采用空间滤波、热致 双折射补偿等技术, 提高激光光束质量, 获得了单脉冲能 量大于 $330 \mathrm{~mJ}$ 、脉宽 $70 \mathrm{ps}$ 、光束质量 $M^{2}<2.5\left(M^{2} x=2.13\right.$ 和 $\left.M^{2} y=2.35\right)$ 的高光束质量皮秒激光输出, 如图1(e)所示.

该大能量高光束质量皮秒激光器将用于地面观测台站 与月球或探月飞行器之间(最远可达 45 万千米)的厘米级高 精度距离探测光源.

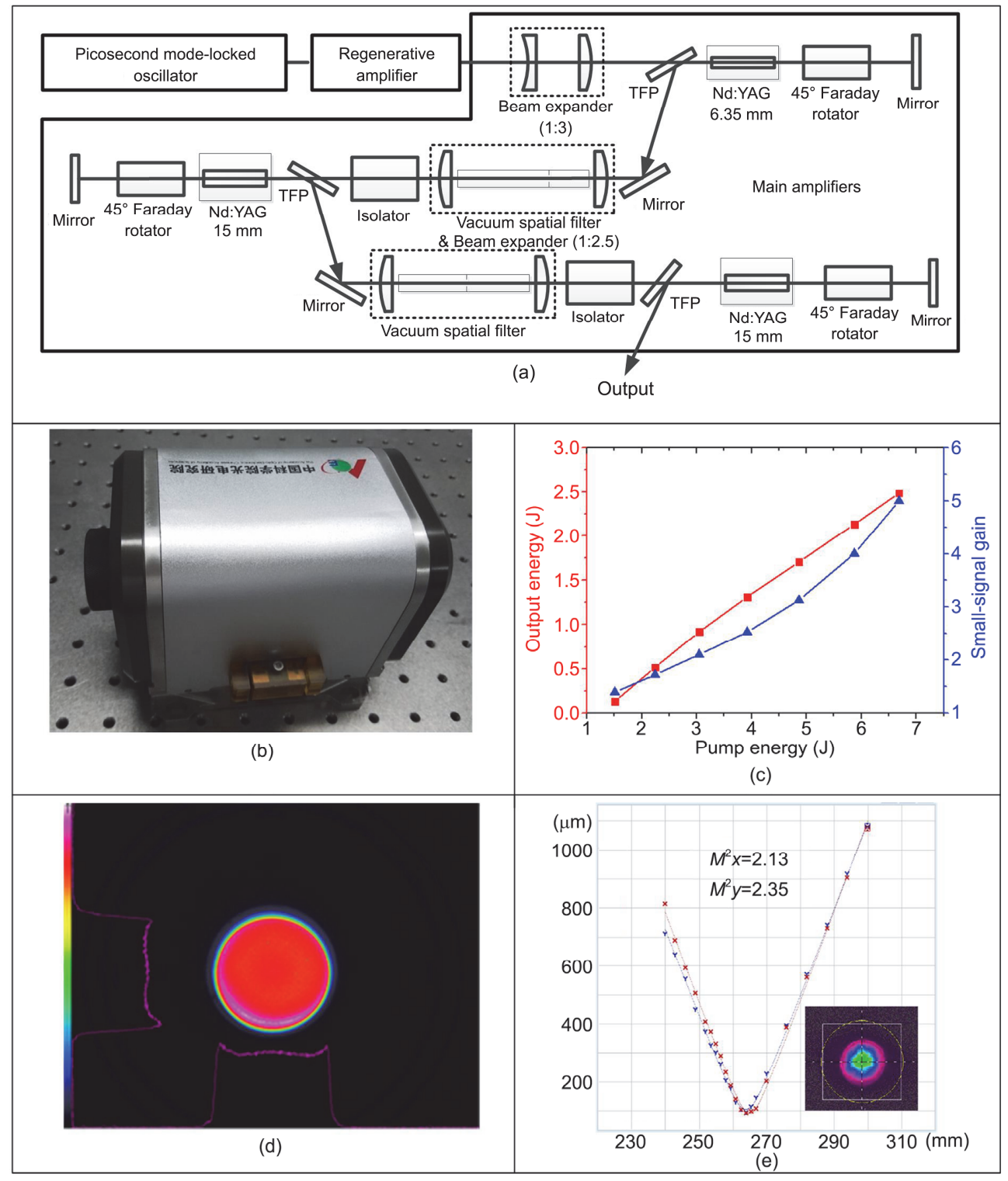

图 1 (网络版彩色)实验装置和关键器件示意图及其主要工作参数. (a) 实验装置示意图; (b) $15 \mathrm{~mm}$ 大口径侧葲 Nd:YAG 模块; (c) Nd:YAG 模块 储能和小信号增益随泵浦能量的变化; (d) 重复频率 $100 \mathrm{~Hz}$ 、泵浦能量 $6.7 \mathrm{~J}$ 时, 泵浦光分布; (e) 脉冲能量 $330 \mathrm{~mJ}$ 时, 激光光束质量 $\left(M^{2} x=2.13\right.$, $\left.M^{2} y=2.35\right)$

Figure 1 (Color online) Schematic of experimental setup and picture of key module with their main working parameters. (a) Schematic of experimental setup; (b) picture of side-pumped Nd:YAG module with rod diameter of $15 \mathrm{~mm}$; (c) output energy and small-signal gain of the module with different pump energy; (d) pump distribution at $100 \mathrm{~Hz}$ and pump energy of $6.7 \mathrm{~J}$; (e) beam quality of $M^{2}$ at output energy of $330 \mathrm{~mJ}$ 